\title{
线性和指数回归方法对土壤呼吸 $\mathrm{CO}_{2}$ 扩散 速率估算的影响
}

\author{
温学发 ${ }^{1}$ 孙晓敏 ${ }^{1}$ 刘允芬 ${ }^{1}$ 李晓波 $^{2}$ \\ (1 中国科学院地理科学与资源研究所 北京 100101) (2 北京力高泰科技有限公司 北京 100035)
}

摘 要 理论上, 土壤呼吸通量的量值可以通过观测土壤呼吸 $\mathrm{CO}_{2}$ 扩散速率 $(\partial c / \partial t)$ 计算得到。但是为获得 $\partial c / \partial t$ 通常须允许土壤呼吸箱内 $\mathrm{CO}_{2}$ 浓度升高, 因此, 如何估算外界大气 $\mathrm{CO}_{2}$ 浓度条件下的 $\partial c / \partial t$ 是土壤呼吸观 测技术的关键，关系到观测结果的准确性。通常 $\partial c / \partial t$ 的估算会受土壤表层大气 $\mathrm{CO}_{2}$ 扩散梯度 (即土壤呼吸箱内 $\mathrm{CO}_{2}$ 扩散梯度和大气 $\mathrm{CO}_{2}$ 浓度昼夜变化)的影响。目前 线性回归方法是土壤呼吸观测中估算 $\partial c / \partial t$ 的基本方法。 然而, 常用的线性回归方法会低估 $\partial c / \partial t$, 而指数回归方法则可以准确地估算 $\partial c / \partial t$ 。夜间 $\partial c / \partial t$ 的变化与大气 $\mathrm{CO}_{2}$ 浓度之间存在非常明显的负相关关系。夜间土壤表层大气 $\mathrm{CO}_{2}$ 扩散梯度的减小导致线性回归方法明显低估 $\partial c / \partial t 。 \partial c / \partial t$ 的昼夜变化过程存在明显的非对称性现象，而指数回归方法可以更好地描述 $\partial c / \partial t$ 昼夜变化的非对 称性响应。

关键词 土壤呼吸 $\mathrm{CO}_{2}$ 扩散梯度 $\mathrm{CO}_{2}$ 扩散速率 线性回归 指数回归 日变化

\section{EFFECTS OF LINEAR AND EXPONENTIAL FITTING ON THE INITIAL RATE OF CHANGE IN $\mathrm{CO}_{2}$ CONCENTRATION ACROSS THE SOIL SURFACE}

\author{
WEN Xue-Fa ${ }^{1}$, SUN Xiao-Min ${ }^{1}$, LIU Yun-Fen ${ }^{1}$, and LI Xiao-Bo ${ }^{2}$ \\ ${ }^{1}$ Institute of Geographic Sciences and Natural Resources Research , Chinese Academy of Sciences , Beijing 100101 , China , and ${ }^{2}$ Beijing Ecotek Tech- \\ nology Company Limited , Beijing 100035 , China
}

\begin{abstract}
Aims Soil $\mathrm{CO}_{2}$ flux is driven primarily by the $\mathrm{CO}_{2}$ diffusion gradient across the soil surface. Ideally , the soil $\mathrm{CO}_{2}$ flux measurement should be made without affecting the diffusion gradient across the soil surface. With the closed chamber system , the soil $\mathrm{CO}_{2}$ diffusion rate $(\partial c / \partial t)$ is required to estimate the soil $\mathrm{CO}_{2}$ flux. To obtain the $\partial c / \partial t$, the chamber $\mathrm{CO}_{2}$ concentration must be allowed to rise. Consequently , the $\partial c / \partial t$ will be affected by the $\mathrm{CO}_{2}$ diffusion gradient across the soil surface because of the decreased $\mathrm{CO}_{2}$ diffusion gradient in the soil chamber. Additionally, the $\partial c / \partial t$ will also be affected by the diurnal variation of the $\mathrm{CO}_{2}$ concentration across the soil surface. Our objective was to compare linear and exponential fitting methods to estimate $\partial c / \partial t$
\end{abstract}

Methods Currently, the $\partial c / \partial t$ is commonly estimated using linear fitting regression. Instead of using the linear fitting method, an exponential fitting method is used to fit the time series of chamber $\mathrm{CO}_{2}$ concentration adopted in the LI-8100 automated soil $\mathrm{CO}_{2}$ flux system.

Important findings The $\partial c / \partial t$ estimated from the linear slopes was consistently underestimated as compared to that from exponential initial slopes. Nighttime $\partial c / \partial t$ was significantly negatively correlated with soil surface $\mathrm{CO}_{2}$ concentration , suggesting that the decreased $\mathrm{CO}_{2}$ diffusion gradient across the soil surface strongly influences the $\partial c / \partial t$. For the closed-chamber method, linear curve fitting significantly underestimated the $\partial c / \partial t$ rate during the nighttime. These results demonstrated the importance of estimating the $\partial c / \partial t$ at ambient soil surface $\mathrm{CO}_{2}$ concentration. The response of the $\partial c / \partial t$ to air temperature exhibited significant asymmetry characteristic , showing that it is a better way for exponential fitting to make long-term and continuous soil $\mathrm{CO}_{2}$ flux measurement to elucidate the magnitudes and processes of soil $\mathrm{CO}_{2}$ flux in the typical terrestrial ecosystem.

Key words soil respiration, $\mathrm{CO}_{2}$ diffusion gradient, $\mathrm{CO}_{2}$ diffusion rate, linear fit, exponential fit , diurnal variation 
目前全球碳循环的研究工作主要是估算各碳库 的储量和碳库间的交换通量。土壤碳库是陆地生态 系统中除岩石圈以外的最大的碳库。Cox 等 (2000) 的模型预测 全球气候变暖正导致生态系统呼吸的 增加，2050 年陆地生态系统将有可能成为碳源。生 态系统呼吸是植物根系、叶片和茎自养呼吸以及土 壤微生物呼吸的总和, 而土壤呼吸则是土壤微生物 和植物根系呼吸的总和。土壤呼吸的量级可高达生 态系统呼吸的 75\% (Goulden et al. , 1996 ; Law et $a l$. , 2002)。生态系统植被冠层碳过程与土壤碳过 程的耦合机制、植物根系自养呼吸和土壤微生物异 养呼吸对环境要素的响应特征、土壤呼吸与地上部 分碳过程和土壤中碳变化的耦合测定、地下部分碳 过程对气候极端事件(如干旱胁迫)或脉冲性降水等 的响应等, 都已经成为全球气候变化研究的热点问 题(Ryan \& Law , 2005 ; Baldocchi , 2005)。迫切需要 长期和连续的土壤呼吸过程的准确观测数据, 以量 化环境和生物要素对土壤呼吸过程的影响, 为土壤 呼吸过程模型的建立和验证提供科学依据，更精确 地阐明土壤呼吸的量级和过程在全球碳循环和碳平 衡中的作用 (Grace \& Rayment, 2000)。

关于土壤呼吸观测的研究始于 80 多年前 (Gainey, 1919)。目前, 国内土壤呼吸观测研究的主 要方法有静态箱/碱液吸收法、静态箱/气象色谱法 和静态箱或动态箱/红外气体分析仪 (IRGA) 法等 (崔骁勇等, 2001; 易志刚和蚁伟民，2003;马秀梅等， 2004)。土壤呼吸量通常是直接测定从土壤表面释 放的 $\mathrm{CO}_{2}$ 浓度变化率 $\left(d \mathrm{CO}_{2} / d t\right)$ 来确定的。理想情 况下土壤呼吸测定应该不影响近地表层大气 $\mathrm{CO}_{2}$ 扩 散梯度, 在这个前提下, 土壤呼吸通量的量值可以通 过观测 $d \mathrm{CO}_{2} / d t$ 的初始斜率 (土壤呼吸 $\mathrm{CO}_{2}$ 扩散速 率, $\partial c / \partial t)$ 计算得到。通常 $\partial c / \partial t$ 的估算会受土壤 表层大气 $\mathrm{CO}_{2}$ 扩散梯度 (即土壤呼吸箱内 $\mathrm{CO}_{2}$ 扩散 梯度和大气 $\mathrm{CO}_{2}$ 浓度昼夜变化) 的影响。如何获得 $d \mathrm{CO}_{2} / \mathrm{dt}$ 的斜率是土壤呼吸观测技术的关键, 因为 这关系到观测结果的准确与否。在实施观测过程中 如果这个问题处理不当, 将导致土壤呼吸测定受到 近地表层大气 $\mathrm{CO}_{2}$ 扩散梯度减小的影响。目前, 大 部分土壤呼吸观测并没有充分考虑到近地表层大气 $\mathrm{CO}_{2}$ 扩散梯度降低对土壤呼吸 $\mathrm{CO}_{2}$ 扩散速率的影 响。为此我们初步探讨以下问题: 1) 土壤呼吸箱内 $\mathrm{CO}_{2}$ 扩散梯度对土壤呼吸 $\mathrm{CO}_{2}$ 扩散速率的影响 ;2) 昼夜 $\mathrm{CO}_{2}$ 扩散梯度变化对土壤呼吸 $\mathrm{CO}_{2}$ 扩散速率的 影响 3)土壤呼吸 $\mathrm{CO}_{2}$ 扩散速率的昼夜变化特征，以
期为精确地阐明土壤呼吸的量级和过程等问题提供 科学依据。

\section{1 材料和方法}

样地设在江西省泰和县中国科学院千烟洲试验 站内 $\left(26^{\circ} 44^{\prime} 52^{\prime \prime} \mathrm{N}, 115^{\circ} 03^{\prime} 47^{\prime \prime} \mathrm{E}\right)$ 的中亚热带人工针 叶林通量站。采用美国 LICOR 公司的 LI-8100 全自 动土壤碳通量测量系统 (Model LI-8100，Licor Inc.； 北京力高泰科技有限公司提供) 测定试验区内不同 条件下的土壤呼吸 $\mathrm{CO}_{2}$ 扩散速率。分 3 种处理 :1) 选取含地表淍落物土壤、去除地表淍落物土壤和土 壤挖壕并去除凋落物土壤。每个处理重复 3 次, 测 定时间为 $2 \mathrm{~min}$ 。2)选取含地表调落物土壤, 土壤呼 吸测定时间分别为 $5 、 10 、 15 、 20 、 25$ 和 $30 \mathrm{~min}$ 。每个 处理重复 3 次。3) 选取林外裸地, 连续 $3 \mathrm{~d}$ 测定土 壤呼吸 $\mathrm{CO}_{2}$ 扩散速率日变化特征。测定时间为 2 $\min$, 每 $30 \mathrm{~min}$ 测定 3 次。

土壤呼吸 $\mathrm{CO}_{2}$ 扩散速率分别采用线性和指数回 归方法进行估算。目前线性回归是大部分土壤呼吸 测量估算土壤呼吸 $\mathrm{CO}_{2}$ 扩散速率 $(\partial c / \partial t$ ) 的基本方 法，

$$
c(t)=c+b t
$$

式中, $c(t)$ 为土壤呼吸箱内 $\mathrm{CO}_{2}$ 的浓度, $c$ 为土壤呼 吸箱关闭时大气 $\mathrm{CO}_{2}$ 浓度, $b$ 为土壤呼吸 $\mathrm{CO}_{2}$ 扩散 速率 $(\partial c / \partial t)$ 。

指数回归方法是美国 LICOR 公司 LI-8100 全自 动碳通量测量系统估算土壤呼吸 $\mathrm{CO}_{2}$ 扩散速率 $(\partial c / \partial t)$ 的主要方法(详见 LI8100 仪器说明书)，

$$
c(t)=c_{x}+\left(c_{0}-c_{x}\right) e^{-a\left(t-t_{0}\right)}
$$

式中, $c(t)$ 为土壤呼吸箱内 $\mathrm{CO}_{2}$ 的浓度, $c_{x}$ 为定义 渐进线的参数, $c_{0}$ 为土壤呼吸箱关闭时大气 $\mathrm{CO}_{2}$ 的 浓度。土壤呼吸 $\mathrm{CO}_{2}$ 扩散速率即为指数回归方程 (2) 的初始斜率 $(\partial c / \partial t)$ :

$$
\partial c / \partial t=a\left(c_{x}-c_{0}\right)
$$

\section{2 结果与讨论}

\section{1 土壤呼吸箱内 $\mathrm{CO}_{2}$ 扩散梯度对土壤呼吸 $\mathrm{CO}_{2}$} 扩散速率的影响

图 1a 描述了土壤呼吸箱内 $\mathrm{CO}_{2}$ 浓度 $120 \mathrm{~s}$ 内的 变化过程。由图 1a 可见, 随着观测时间的增加, 土 壤呼吸箱内 $\mathrm{CO}_{2}$ 浓度逐渐增加并导致土壤呼吸箱内 $\mathrm{CO}_{2}$ 扩散梯度减小, 这将会抑制土壤 $\mathrm{CO}_{2}$ 的释放并 降低土壤呼吸 $\mathrm{CO}_{2}$ 扩散速率。为此, 准确估算 $d \mathrm{CO}_{2} / d t$ 的初始斜率获得土壤呼吸 $\mathrm{CO}_{2}$ 扩散速率 
$(\partial c / \partial t)$ 是非常重要的, 它关系到观测结果的准确 与否。

目前,线性回归方法是土壤呼吸测量中估算 $d \mathrm{CO}_{2} / d t$ 的初始斜率的基本途径。而基于公式 (2) 和(3)的指数回归方法将是计算 $d \mathrm{CO}_{2} / d t$ 的初始斜 率的新的有效途径。由图 $1 \mathrm{a}$ 可以看出, 指数回归方 法可以更加准确地拟合土壤呼吸箱内 $\mathrm{CO}_{2}$ 浓度的变 化，从而准确地估算 $\partial c / \partial t$ 。图 $1 \mathrm{~b}$ 对比分析了指数 和线性回归方法对不同土壤呼吸处理的土壤呼吸 $\mathrm{CO}_{2}$ 扩散速率估算的影响。由图 $1 \mathrm{~b}$ 可以看出, 虽然 线性回归与指数回归方法估算的 $\partial c / \partial t$ 具有很好的 线性关系, 但是线性回归获得的 $\partial c / \partial t$ 低于指数回 归估算的 $\partial c / \partial t$ 的大约 $11 \%$, 这将导致土壤呼吸量 的显著低估。
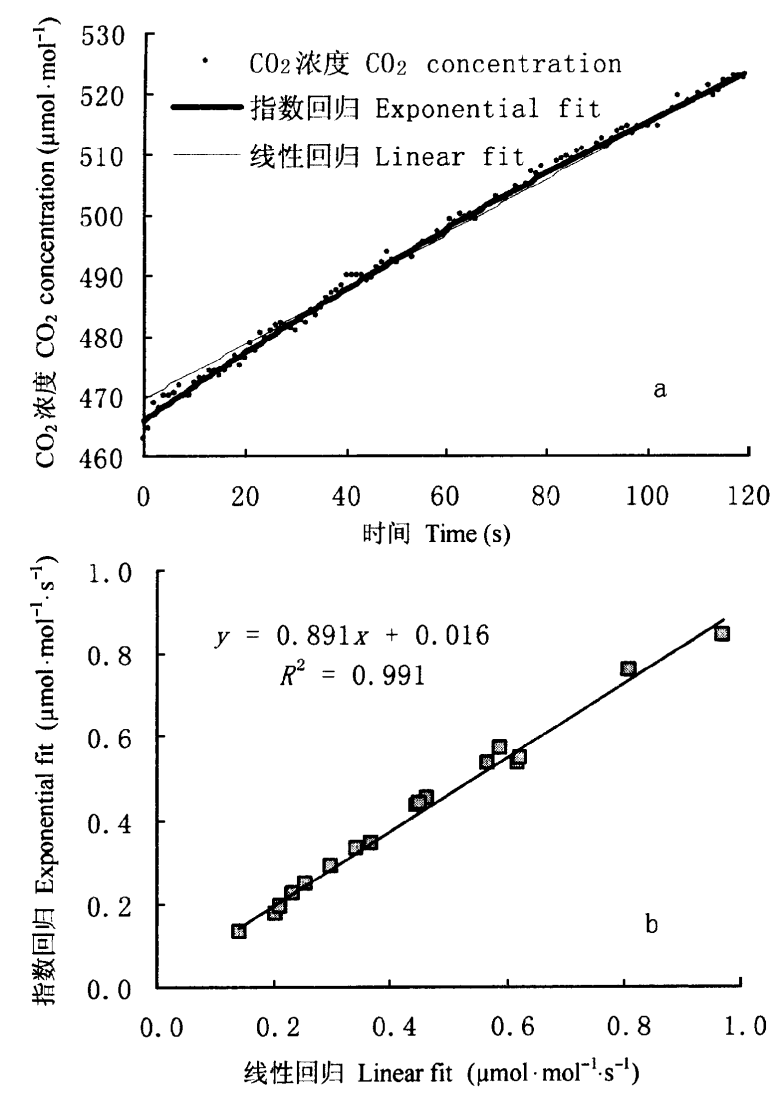

图 1 指数和线性回归方法对土壤呼吸 $\mathrm{CO}_{2}$ 扩散速率 $(\partial c / \partial t)$ 的影响 Fig. 1 Impacts of exponential and linear fit on the initial rate of change in the $\mathrm{CO}_{2}$ concentration across the soil surface

$\mathrm{a}:$ 土壤呼吸箱内 $\mathrm{CO}_{2}$ 浓度的变化过程 Variations of $\mathrm{CO}_{2}$ concentration in the soil respiration chamber $\mathrm{b}$ : 材料与方法中试验处理 1) Experiment 1) described in Material and Methods

图 2 对比分析了土壤呼吸测定时间分别为 5 、 $10 、 15 、 20 、 25$ 和 $30 \mathrm{~min}$ 条件下线性回归和指数回归 方法对土壤呼吸 $\mathrm{CO}_{2}$ 扩散速率的影响。可以看出,
随着土壤呼吸测定时间的增加, 由于土壤呼吸箱内 $\mathrm{CO}_{2}$ 扩散梯度的降低导致线性回归方法估算的 $\partial c /$ $\partial t$ 呈现逐渐降低的趋势, 而指数回归方法估算的 $\partial c / \partial t$ 仍然保持相对稳定。然而, 目前大部分土壤 呼吸观测并没有充分考虑到土壤呼吸箱内 $\mathrm{CO}_{2}$ 扩散 梯度降低对土壤呼吸的影响，而是仅仅采用线性回 归方法估算 $\partial c / \partial t$ 。指数回归方法可以解决土壤呼 吸箱内 $\mathrm{CO}_{2}$ 扩散梯度减小对 $\partial c / \partial t$ 估算的影响。但 是需要指出的是, 如果观测时间过长还会导致温度 等其它环境要素的改变，因此，观测时间应尽可能缩 短。

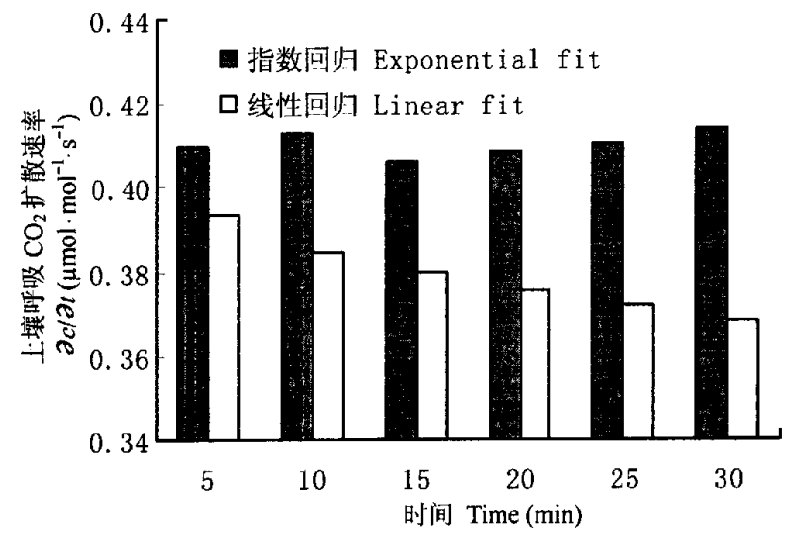

图 2 测量时间对土壤呼吸 $\mathrm{CO}_{2}$ 扩散速率 $(\partial c / \partial t)$ 的影响

Fig.2 Impacts of different measuring periods on the initial rate of change in $\mathrm{CO}_{2}$ concentration $(\partial c / \partial t)$ across the soil surface

2.2 昼夜 $\mathrm{CO}_{2}$ 扩散梯度变化对土壤呼吸 $\mathrm{CO}_{2}$ 扩散 速率的影响

图 3 描述了指数和线性回归估算的土壤呼吸 $\mathrm{CO}_{2}$ 扩散速率和大气 $\mathrm{CO}_{2}$ 浓度的日变化特征。由图 3 可见,大气 $\mathrm{CO}_{2}$ 浓度与指数和线性回归估算的 $\partial c / \partial t$ 都具有明显的日变化特征，但是具有相反的 趋势。从图 3 也可看出, 当夜间大气 $\mathrm{CO}_{2}$ 浓度较高 时，即土壤表层大气 $\mathrm{CO}_{2}$ 扩散梯度较小时，指数回归 与线性回归所获得的 $\partial c / \partial t$ 的差异非常明显, 而白 天大气 $\mathrm{CO}_{2}$ 浓度较低即土壤表层大气 $\mathrm{CO}_{2}$ 扩散梯度 较大时 , 二者的差异较小。

根据太阳高度角的大小, 可以将图 3 中指数和 线性回归估算的土壤呼吸 $\mathrm{CO}_{2}$ 扩散速率和大气 $\mathrm{CO}_{2}$ 浓度的日变化数据分为白天与夜间两组进行分析。 图 4 描述了白天和夜间土壤表层大气 $\mathrm{CO}_{2}$ 扩散梯度 变化对土壤呼吸 $\mathrm{CO}_{2}$ 扩散速率的影响。从图 4a 可 以看出，除了黎明时的数据外，白天 $\partial c / \partial t$ 与大气 $\mathrm{CO}_{2}$ 浓度关系的斜率较大, 这可能主要是由于白天 
温度变异较大而土壤表层大气 $\mathrm{CO}_{2}$ 浓度相对保持稳 定造成的。白天指数回归和线性回归估算的 $\partial c / \partial t$ 没有显著差异 如图 4c 所示只差 $2.4 \%$ 左右。夜间
土壤呼吸释放的 $\mathrm{CO}_{2}$ 在近地表层积累导致大气 $\mathrm{CO}_{2}$ 浓度升高, 而较高的土壤表层大气 $\mathrm{CO}_{2}$ 浓度抑制了 土壤 $\mathrm{CO}_{2}$ 的释放。夜间 $\partial c / \partial t$ 与土壤表层大气

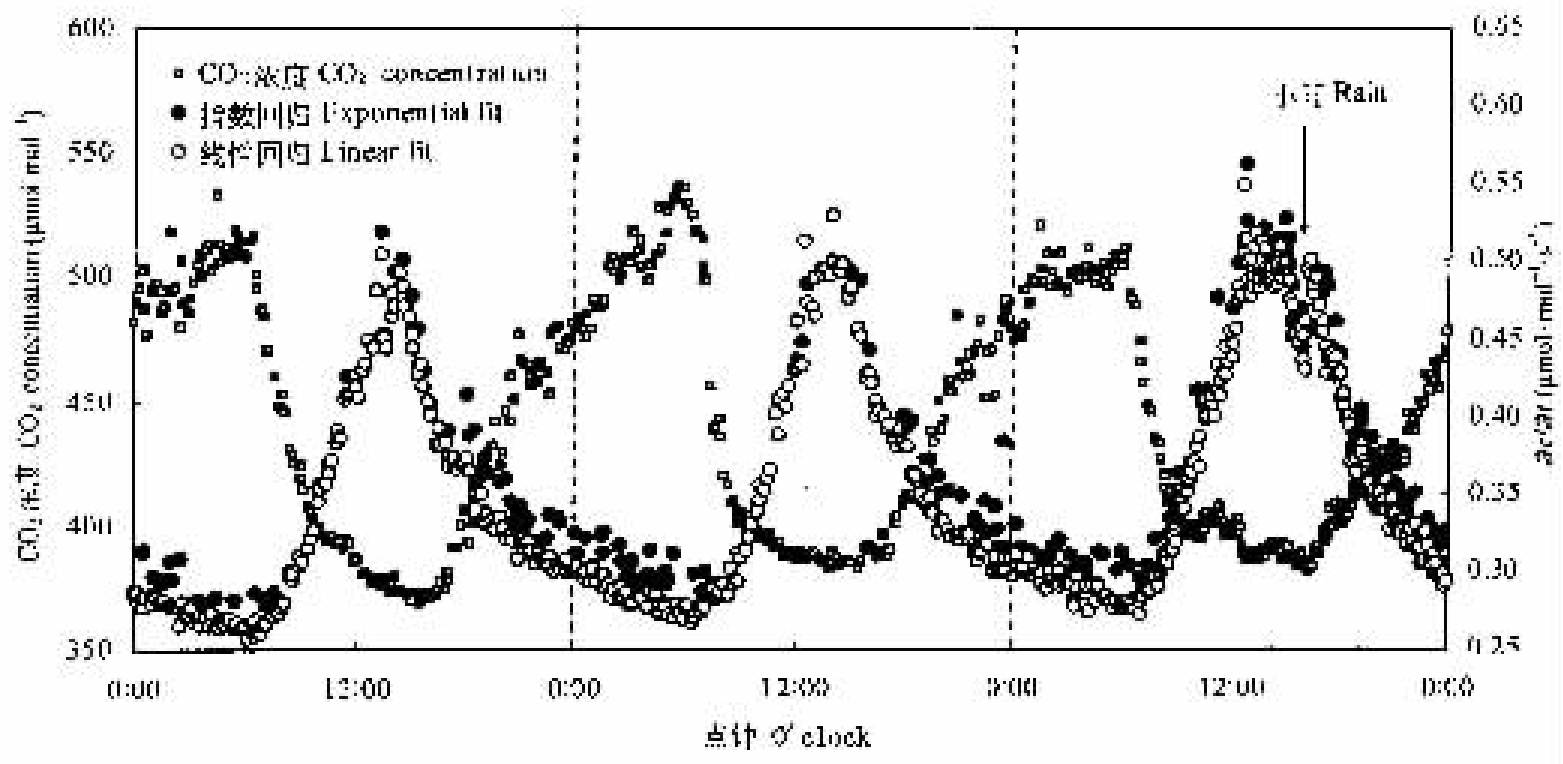

图 3 指数和线性回归估算的土壤呼吸 $\mathrm{CO}_{2}$ 扩散速率 $(\partial c / \partial t)$ 和大气 $\mathrm{CO}_{2}$ 浓度的日变化特征

Fig.3 Diurnal variation of $\mathrm{CO}_{2}$ concentration and the initial rate of change in the $\mathrm{CO}_{2}$ concentration $(\partial c / \partial t)$ by the exponential and linear fit across the soil surface
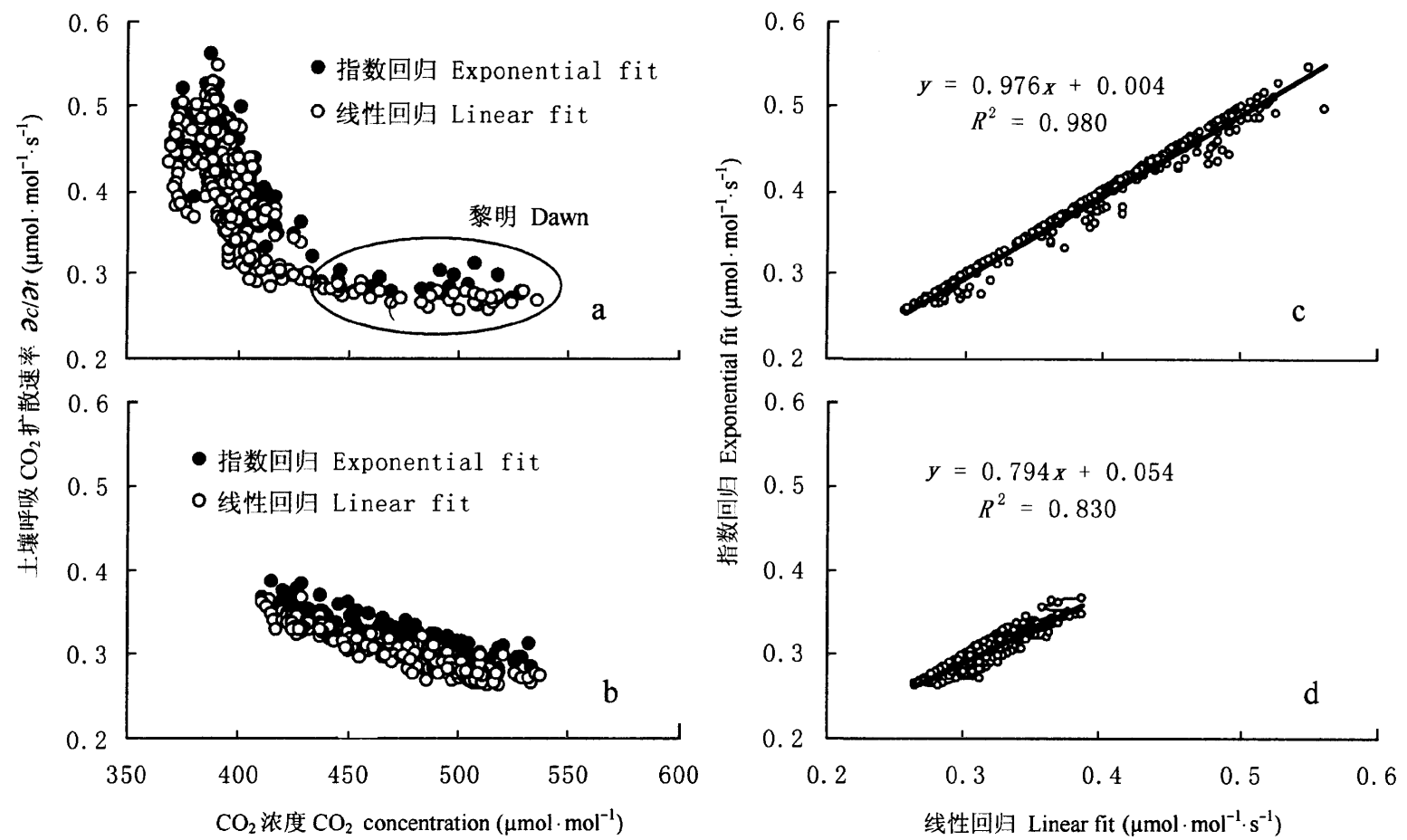

图 4 昼夜 $\mathrm{CO}_{2}$ 扩散梯度变化对土壤呼吸 $\mathrm{CO}_{2}$ 扩散速率 $(\partial c / \partial t)$ 的影响

Fig.4 Impact of the diurnal $\mathrm{CO}_{2}$ diffusion gradient on the initial rate of change in $\mathrm{CO}_{2}$ concentration $(\partial c / \partial t)$ across the soil surface $\mathrm{a}$ 和 $\mathrm{c}$ 指白天数据 $\mathrm{a}$ and $\mathrm{c}$ refer to daytime data $\mathrm{b}$ 和 $\mathrm{d}$ 指夜间数据 $\mathrm{b}$ and $\mathrm{d}$ refer to nighttime data 
$\mathrm{CO}_{2}$ 浓度之间存在非常明显的负相关关系(图 4b)。 夜间指数回归和线性回归估算的 $\partial c / \partial t$ 之间差异可 高达 20.6\% (图 4d) ,这表明线性回归估算 $\partial c / \partial t$ 将 会导致夜间土壤呼吸量的低估。指数回归方法是解 决估算外界土壤表层大气 $\mathrm{CO}_{2}$ 扩散梯度时的 $\partial c / \partial t$ 的重要方法之一, 可以最大限度地避免昼夜 $\mathrm{CO}_{2}$ 扩 散梯度变化对准确估算 $\partial c / \partial t$ 的影响。

2.3 土壤呼吸 $\mathrm{CO}_{2}$ 扩散速率昼夜变化的非对称性 现象

图 5 描述了土壤呼吸 $\mathrm{CO}_{2}$ 扩散速率对温度昼夜 变化响应的非对称响应特征。从图 5 可以看出, 上 午与下午的温度变化对 $\partial c / \partial t$ 的影响明显不同, 表 现出 $\partial c / \partial t$ 的昼夜变化存在明显的非对称性现象， 指数回归方法可以更好地描述 $\partial c / \partial t$ 昼夜变化的非
对称性响应特征。对比图 $5 \mathrm{a} 、 5 \mathrm{~b}$ 与 $5 \mathrm{c}$ 可以发现, 图 $5 c$ 中 $\partial c / \partial t$ 对温度昼夜变化响应的非对称性响应 特征有所变化, 这主要是由于图 $5 \mathrm{c}$ 的日变化的下午 数据受到小雨的影响, 导致 $\partial c / \partial t$ 迅速上升(图 3)。 降水对土壤呼吸的影响因时因地而异。脉冲性降水 事件会强烈地激发土壤 $\mathrm{CO}_{2}$ 的释放(图 3)。

目前, 大部分土壤呼吸观测通常是用 9:00 和 12:00之间的测定值代表日呼吸量的平均值 (Davidson et al ., 1998 ; Yan et al .,2006)。但是这些观测无法 很好地描述土壤呼吸的非对称性响应过程, 必然会对 土壤呼吸过程模型的建立和验证带来一定的影响与 选择性系统误差。总之, 为精确地阐明土壤呼吸的量 级和过程在全球碳循环和碳平衡中的作用, 非常必要 进行长期和连续的准确的土壤呼吸观测。

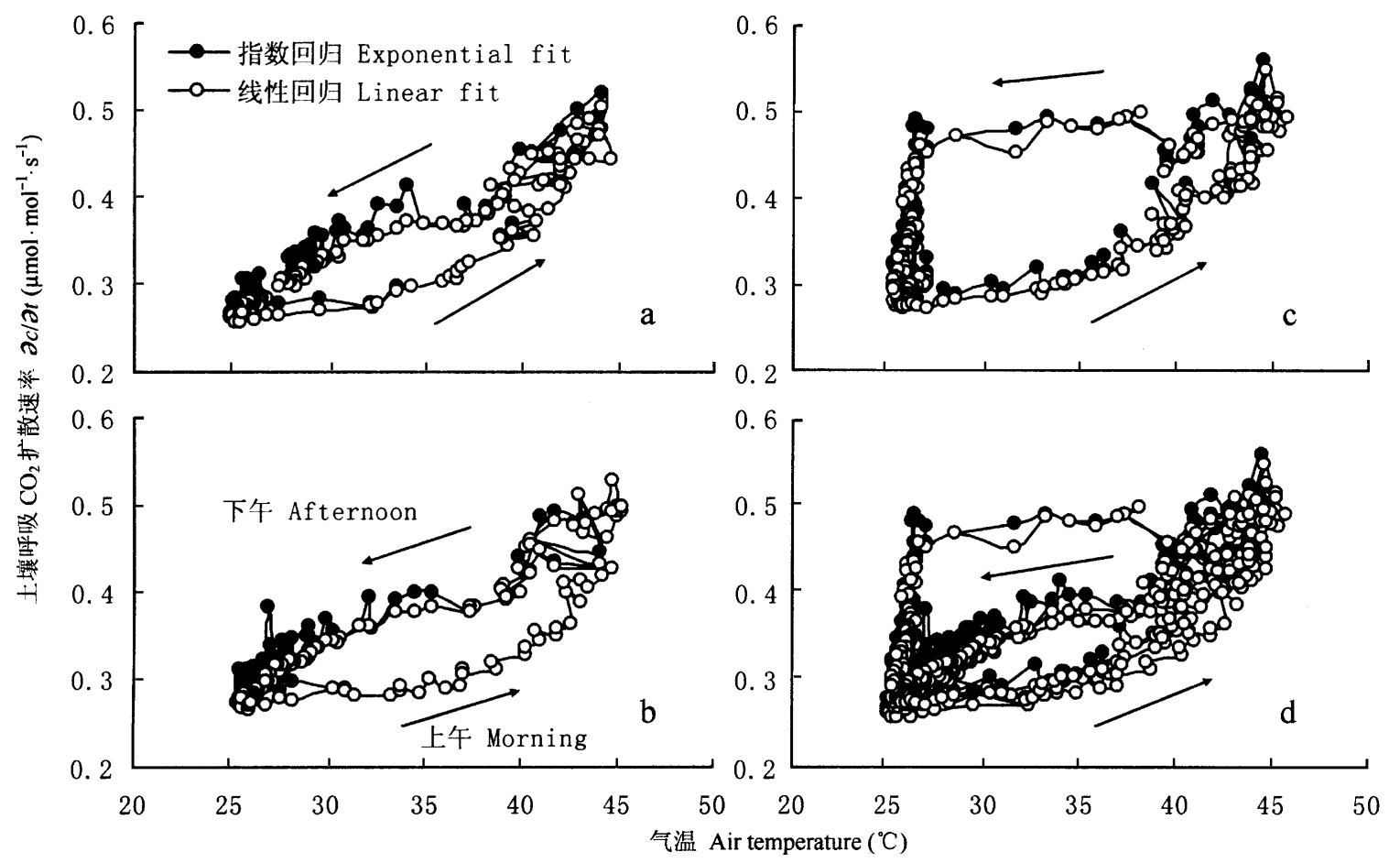

图 5 土壤呼吸 $\mathrm{CO}_{2}$ 扩散速率 $(\partial c / \partial t)$ 对温度昼夜变化响应的非对称性响应特征

Fig.5 Diurnal asymmetry of the initial rate of change in $\mathrm{CO}_{2}$ concentration $(\partial c / \partial t)$ in response to air temperature across the soil surface $\mathrm{a} 、 \mathrm{~b}$ 和 $\mathrm{c}$ 分别代表图 3 中第一天、第二天和第三天的日变化数据 $\mathrm{a}, \mathrm{b}$ and $\mathrm{c}$ refer to the data on the 1st, 2nd and 3rd day , respectively , in Fig. 3 $\mathrm{d}$ 代表图 3 中所有的日变化数据 $\mathrm{d}$ refers to all data in Fig. 3

\section{3 结 论}

理想情况下, 土壤呼吸测定应该不影响土壤 $\mathrm{CO}_{2}$ 扩散梯度, 在这个前提下, 土壤呼吸通量的量值 可以通过观测 $d \mathrm{CO}_{2} / d t$ 的初始斜率 (土壤呼吸 $\mathrm{CO}_{2}$ 扩散速率)计算得到。通常土壤呼吸 $\mathrm{CO}_{2}$ 扩散速率
的估算会受到土壤表层大气 $\mathrm{CO}_{2}$ 扩散梯度 (即土壤 呼吸箱内 $\mathrm{CO}_{2}$ 扩散梯度和大气 $\mathrm{CO}_{2}$ 浓度昼夜变化) 的影响。为获得 $d \mathrm{CO}_{2} / d t$ 的初始斜率, 须允许土壤 呼吸箱内 $\mathrm{CO}_{2}$ 浓度升高, 但土壤呼吸箱内的 $\mathrm{CO}_{2}$ 浓 度逐渐增加会导致土壤呼吸箱内 $\mathrm{CO}_{2}$ 扩散梯度减 小。线性回归方法获得的土壤呼吸 $\mathrm{CO}_{2}$ 扩散速率低 
于指数回归的估算值, 而指数回归方法可以更加准 确地估算土壤呼吸 $\mathrm{CO}_{2}$ 扩散速率。夜间土壤呼吸 $\mathrm{CO}_{2}$ 扩散速率与土壤表层大气 $\mathrm{CO}_{2}$ 浓度之间存在非 常明显的负相关关系,表明夜间土壤表层大气 $\mathrm{CO}_{2}$ 扩散梯度的降低显著抑制土壤呼吸 $\mathrm{CO}_{2}$ 扩散速率的 增加。土壤呼吸 $\mathrm{CO}_{2}$ 扩散速率昼夜变化具有明显的 非对称性现象 指数回归方法可以更好地描述土壤 呼吸 $\mathrm{CO}_{2}$ 扩散速率昼夜变化的非对称性响应特征。 为阐明土壤呼吸的量级和过程在全球碳循环和碳平 衡中的作用，进行长期和连续的准确的土壤呼吸观 测是非常必要的。

\section{参 考 文 献}

Baldocchi D (2005). The carbon cycle under stress. Nature, 437, $483-484$.

Cox PM, Betts RA, Jones CD, Spall SA, Totterdell IJ (2000). Acceleration of global warming due to carbon-cycle feedbacks in a coupled climate model. Nature, 408, $184-187$.

Cui XY (崔骁勇)，Chen ZZ (陈佐忠)，Chen SQ (陈四清) (2001). Progress in research on soil respiration of grasslands. Acta Ecologica Sinica (生态学报), 21, 315-325. (in Chinese with English abstract)

Davidson EA, Belk E, Boone RD (1998). Soil water content and temperature as independent or confounded factors controlling soil respiration in a temperate mixed hardwood forest. Global Change
Biology, 4, $217-227$.

Gainey PL (1919) . Parallel formation of carbon dioxide, ammonia and nitrate in soil. Soil Science, 7, $293-311$.

Goulden ML, Munger JW, Fan SM, Daube BC, Wofsy SC (1996) . Measurements of carbon sequestration by long-term eddy covariance: methods and a critical evaluation of accuracy. Global Change Biology, 2, $169-182$.

Grace J, Rayment M (2000). Respiration in the balance. Nature, $404,819-820$.

Law BE, Falge E, Gu L, Baldocchi D (2002). Environmental controls over carbon dioxide and water vapor exchange of terrestrial vegetation. Agricultural and Forest Meteorology, 113, 97 - 120 .

Ma XM (马秀梅), Zhu B (朱波), Han GX (韩广轩), Chen YC (陈玉成), Gao MR (高美荣), Zhang ZJ (张中杰) (2004). The advance in research of soil respiration. Advance in Earth Sciences (地球科学进展), 19, 491 - 495. (in Chinese with English abstract)

Ryan MG, Law BE (2005) . Interpreting, measuring, and modeling soil respiration. Biogeochemistry, 73, 3-27.

Yan JH, Wang YP, Zhou GY, Zhang DQ (2006) . Estimates of soil respiration and net primary production of three forests at different succession stages in South China. Global Change Biology, 12, $810-821$.

Yi ZG (易志刚), Yi WM (蚁伟民) (2003). Proceeding of studies on soil respiration of forest ecosystem. Ecology and Environment (生态环境), 12, 361 - 365. (in Chinese with English abstract) 